\title{
BMJ Open Feasibility of using administrative data for identifying medical reasons to delay hip fracture surgery: a Canadian database study
}

\author{
Pierre Guy, ${ }^{1}$ Katie J Sheehan, ${ }^{2}$ Suzanne N Morin, ${ }^{3}$ James Waddell, ${ }^{4}$ \\ Michael Dunbar, ${ }^{5}$ Edward Harvey, ${ }^{6}$ Susan Sirett, ${ }^{7}$ Boris Sobolev, ${ }^{8}$ Lisa Kuramoto, ${ }^{9}$ \\ Michael Tang ${ }^{9}$ for the Canadian Collaborative Study on Hip Fractures
}

To cite: Guy P, Sheehan KJ, Morin SN, et al. Feasibility of using administrative data for identifying medical reasons to delay hip fracture surgery: a Canadian database study. BMJ Open 2017;7:e017869. doi:10.1136/ bmjopen-2017-017869

- Prepublication history and additional material for this paper are available online. To view please visit the journal (http:// dx.doi.org/10.1136/bmjopen2017-017869).

Received 22 May 2017 Revised 26 July 2017 Accepted 23 August 2017

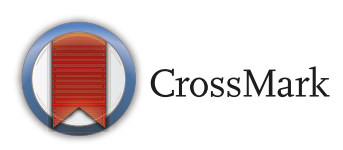

For numbered affiliations see end of article.

Correspondence to

Dr Katie J Sheehan;

katie.sheehan@kcl.ac.uk

\section{ABSTRACT}

Purpose Failure to account for medically necessary delays may lead to an underestimation of early surgery benefits. This study investigated the feasibility of using administrative data to identify the National Institute for Health and Care Excellence (NICE) 124 guideline list of conditions that appropriately delay hip fracture surgery. Methods We assembled a list of diagnosis and procedure codes to reflect the NICE 124 conditions. The list was reviewed and updated by an advanced clinical coder. The list was refined by five clinical experts. We then screened Canadian Institute for Health Information discharge abstracts for 153918 patients surgically treated for a non-pathological first hip fracture between 1 January 2004 and 31 December 2012 for diagnosis codes present on admission and procedure codes that antedated hip fracture surgery. We classified abstracts as having medical reasons for delaying surgery based on the presence of these codes.

Results In total, 10237 (6.7\%; 95\% Cl 6.5\% to 6.8\%) patients had diagnostic and procedure codes indicating medical reasons for delay. The most common reasons for medical delay were exacerbation of a chronic chest condition (35.9\%) and acute chest infection (23.2\%). The proportion of patients with reasons for medical delays increased with time from admission to surgery: 3.9\% (95\% Cl 3.6\% to 4.1\%) for same day surgery; $4.7 \%$ (95\% Cl $4.5 \%$ to $4.8 \%$ ) for surgery 1 day after admission; $7.1 \%$ (95\% $\mathrm{Cl} 6.9 \%$ to $7.4 \%)$ for surgery 2 days after admission; and $15.5 \%$ (95\% $\mathrm{Cl} 15.1 \%$ to $16.0 \%)$ for surgery more than 2 days after admission. The trend was seen for admissions on weekday working hours, weekday after hours and on weekends.

Conclusion Administrative data can be considered to identify conditions that appropriately delay hip fracture surgery. Accounting for medically necessary delays can improve estimates of the effectiveness of early surgery.

\section{INTRODUCTION}

Hip fractures occur in older adults as frequently as common cancers but with substantially worse outcomes. ${ }^{1}$ Most patients undergo surgery to improve survival, restore
Strengths and limitations of this study

- This study includes all hospital records of hip fracture surgeries performed in Canada over an 8-year period, as captured by an administrative database.

- The authors assembled the first comprehensive list of diagnosis and procedure codes to identify appropriate medical reasons for delaying hip fracture surgery.

- The list includes the National Institute for Health and Care Excellence 124 guideline conditions; other medical conditions may appropriately delay surgery.

- A chart review may be a better source for studying the prevalence of medical, non-medical and personal reasons for delay but is limited in the number of patients who could be evaluated.

mobility and potentially return to active, independent living. ${ }^{2}$ However, surgical delays may diminish the therapeutic benefits of the surgical procedure by increasing patients' exposure to immobilisation and generalised inflammatory state. ${ }^{3}$ This may lead to potentially fatal thromboembolic, cardiovascular and infectious complications. ${ }^{45}$

Previous research provides inconsistent evidence on the survival benefit of early surgery for hip fracture with reports of increased $^{67}$ and no increased ${ }^{8-11}$ risk of inhospital death with longer waits. An important limitation of this literature lies in the lack of information on the reasons for delay. Delays may result from a lack of hospital resources or issues related to healthcare delivery, broadly referred to as non-medical delays. ${ }^{12}$ On the other hand, the UK National Institute for Health and Care Excellence (NICE) 124 guideline recognises 'there are sometimes legitimate reasons for delay' and lists conditions requiring correction preoperatively. ${ }^{13}$ The 
guideline's experts noted that "Provided these problems are sought and measures initiated to correct them are taken promptly the majority [of patient presenting with hip fractures] can be optimised within 24 hours." 13 That is, some conditions requiring correction preoperatively may cause medically necessary surgical delays for at least one inpatient day.

Failure to differentiate patients with medically necessary and non-medical delays may lead to an underestimation of the benefit of early surgery. Siegmeth et al and Orosz et al suggest excluding unfit patients from timingoutcome analysis on the premise that patients with medically necessary delays may be less likely to die with longer wait times than if they received early surgery. ${ }^{1415}$ However, medical reasons for delaying hip fracture surgery are not readily available in administrative databases. Therefore, this study sought to determine the feasibility of identifying NICE 124 conditions from administrative discharge abstracts through the creation of specific algorithms. ${ }^{13}$

\section{METHODS}

\section{Data source}

We obtained Canadian Institute for Health Information (CIHI) discharge abstracts for all patients 65 years or older who were surgically treated for non-pathological first hip fracture between 1 January 2004 and 31 December 2012 in all Canadian hospitals, except for the province of Quebec. The abstracts were selected using procedure codes for hip fracture surgery (Canadian Classification of Health Intervention (CCI): 1VA $74^{\wedge} \wedge, 1 \mathrm{VA} 53^{\wedge} \wedge, 1 \mathrm{VC} 74^{\wedge}$, 1SQ53^^). Multiple abstracts linked by hospital transfers for the same patient were combined in one care episode. ${ }^{16}$ The University of British Columbia Behavioural Research Ethics Board approved this study.

\section{Diagnostic and procedures codes for NICE 124 conditions}

We initially assembled a list of International Classification of Disease Canadian 10th Revision (ICD-10-CA) diagnosis codes and CCI codes matching conditions from the NICE 124 guideline (KS) ${ }^{13}$ These include anaemia, anticoagulation, volume depletion, electrolyte imbalance, uncontrolled diabetes, uncontrolled heart failure, acute cardiac arrhythmia or ischaemia, acute chest infection or exacerbation of a chronic chest condition. ${ }^{13}$ The list was reviewed and updated by an advanced clinical coder (SS). Subsequently five clinical experts (PG, JW, SM, EH, $\mathrm{MD})$ refined the list of the codes to ensure capturing acute aspect of the NICE 124 conditions (eg, 'exacerbation of chronic chest condition' was linked to code for 'chronic obstructive pulmonary disease with acute lower respiratory infection'). Further, 'anaemia' was linked to code for 'transfusion'. We also identified all codes for complications of the NICE 124 conditions (eg, 'correctable cardiac ischaemia' was linked to code for 'acute myocardial infarction'). We included ICD-10-CA and CCI codes approved by at least one clinical expert (see online supplementary file 1 ).
Box 1 Identifying medically necessary reasons for delaying hip fracture surgery

- Created algorithms of diagnostic and procedures codes associated with National Institute for Health and Care Excellence 124 conditions.

- Screened discharge abstracts for diagnostic codes present on admission and for procedures codes that antedated hip fracture surgery.

- Classified discharge abstracts as having medically necessary reasons for delaying surgery.

\section{Classifying $\mathrm{CIHI}$ discharge abstracts}

We screened CIHI discharge abstracts for ICD-10-CA codes present on admission (type 1 diagnosis) and CCI codes that antedated hip fracture surgery. Diagnoses and procedure codes are included in the CIHI discharge abstracts as mandatory data elements to ensure national coverage. ${ }^{17}$ We then classified the abstracts as having medical reasons for delaying surgery based on the presence of diagnostic codes only, diagnosis or procedure codes or diagnosis and procedure codes (box1). Specifically, we used diagnosis codes only for uncontrolled heart failure and acute chest infection; diagnosis or procedure codes for exacerbation of chronic chest conditions, correctable cardiac ischaemia, correctable cardiac arrhythmia, volume depletion and anticoagulation; and diagnosis and procedure codes for uncontrolled diabetes, anaemia and electrolyte imbalance (see online supplementary file 1). NICE 124 conditions include acute aspects of some chronic conditions, namely, diabetes, heart failure, cardiac ischaemia and cardiac arrhythmia. Classification of abstracts as having chronic conditions and acute aspects of those conditions do not need to be mutually exclusive.

\section{Statistical analysis}

Detailed description of the study population is available elsewhere. ${ }^{18}$ We summarised baseline characteristics of the patient population using descriptive statistics. We estimated the prevalence of medically necessary delays by dividing the number of patients with diagnosis or procedure codes pointing to medical delays by the total number of surgically treated patients, multiplied by $100 \%$, overall and by timing of surgery. Lastly, we estimated the prevalence of medically necessary delays in relation to timing of admission by timing of surgery.

\section{RESULTS}

\section{Patient characteristics}

A total of 153918 patients were surgically treated for a non-pathological first hip fracture between 1 January 2004 and 31 December 2012. Most patients were women $(73.4 \%)$, and almost half were 85 years or older $(45.6 \%)$. Most patients were admitted from home without major comorbidity (heart failure, chronic obstructive pulmonary disease, ischaemic heart disease, hypertension, cardiac arrhythmia or diabetes) $(43.6 \%)$. 
Table 1 Specific National Institute for Health and Care Excellence 124 guideline conditions pointing to possible medical reason for delay to hip fracture surgery among surgically treated patients

\begin{tabular}{|c|c|c|c|}
\hline Condition & Patients (n) & $\%$ & $95 \% \mathrm{Cl}$ \\
\hline Anaemia & 198 & 0.1 & (0.1 to 0.1$)$ \\
\hline Anticoagulation reversal & 495 & 0.3 & (0.3 to 0.3$)$ \\
\hline Volume depletion & 1200 & 0.8 & (0.7 to 0.8$)$ \\
\hline Electrolyte imbalance & 38 & 0.0 & (0.0 to 0.0$)$ \\
\hline Uncontrolled diabetes & 1497 & 1.0 & (0.9 to 1.0$)$ \\
\hline $\begin{array}{l}\text { Uncontrolled heart } \\
\text { failure }\end{array}$ & 26 & 0.0 & (0.0 to 0.0$)$ \\
\hline $\begin{array}{l}\text { Correctable cardiac } \\
\text { arrhythmia }\end{array}$ & 796 & 0.5 & (0.5 to 0.6$)$ \\
\hline $\begin{array}{l}\text { Correctable cardiac } \\
\text { ischaemia }\end{array}$ & 1668 & 1.1 & (1.0 to 1.1$)$ \\
\hline Acute chest infection & 2375 & 1.5 & (1.5 to 1.6$)$ \\
\hline $\begin{array}{l}\text { Exacerbation of chronic } \\
\text { chest condition }\end{array}$ & 3679 & 2.4 & (2.3 to 2.5 ) \\
\hline At least one condition & 10237 & 6.7 & (6.5 to 6.8$)$ \\
\hline
\end{tabular}

Reasons for medical delay

Overall, 10237 (6.7\%; 95\% CI $6.5 \%$ to $6.8 \%)$ surgically treated patients had diagnostic and procedure codes indicating possible medical reasons for delaying surgery (table 1). Most patients had only one medical reason for delay $(84.9 \%)$. The most common reasons for medical delay were exacerbation of a chronic chest condition $(35.9 \%)$, acute chest infection $(23.2 \%)$, correctable cardiac ischaemia $(16.3 \%)$, uncontrolled diabetes $(14.6 \%)$ and volume depletion $(11.7 \%)$.

The prevalence of medical reasons for delay among surgically treated patients increased with the time from admission to surgery: $3.9 \%$ (95\% CI $3.6 \%$ to $4.1 \%$ ) for same day surgery; $4.4 \%$ (95\% CI $4.3 \%$ to $4.5 \%$ ) for surgery within 1 day of admission; and $5.1 \%$ (95\% CI $4.9 \%$ to $5.2 \%$ ) for patients who underwent surgery within 2 days of admission. The prevalence of medical reasons for delay on each surgical day since admission also increased: $3.9 \%$
(95\% CI $3.6 \%$ to $4.1 \%$ ) for same day surgery; $4.7 \%(95 \%$ CI $4.5 \%$ to $4.8 \%$ ) for surgery 1 day after admission; $7.1 \%$ (95\% CI $6.9 \%$ to $7.4 \%$ ) for surgery 2 days after admission; and $15.5 \%$ (95\% CI $15.1 \%$ to $16.0 \%$ ) for surgery more than 2 days after admission. The trend was seen for admissions on weekday working hours, weekday after hours and on weekends (table 2 ).

The prevalence of medical reasons for delay among surgically treated patients decreased with age, from $7.1 \%$ $(95 \%$ CI $6.8 \%$ to $7.5 \%)$ in patients $65-74$ years of age to $5.7 \%(95 \%$ CI $5.2 \%$ to $6.2 \%)$ in those 95 years or older. More men had a medical reason for delay $(9.4 \%$; $95 \%$ CI $9.1 \%$ to $9.7 \%)$ than women $(5.7 \%$; $95 \%$ CI $5.5 \%$ to $5.8 \%)$. The prevalence of medical reasons for delay among surgically treated patients was lowest among patients admitted from home without comorbidities $(2.2 \%$; 95\% CI $2.0 \%$ to $2.3 \%$ ) and highest among those admitted from home with comorbidity or home care $(18.0 \%$; $95 \%$ CI $17.5 \%$ to $18.4 \%$ ). The prevalence of medical reasons for delay among surgically treated patients admitted from longterm care was about one-third lower than those admitted from home with comorbidity or home care $(6.2 \%$; $95 \%$ CI $6.0 \%$ to $6.5 \%$ ).

\section{DISCUSSION}

\section{Main findings}

Overall, $6.7 \%$ of surgically treated patients had a medical condition that may necessitate delay to hip fracture surgery according to the NICE 124 guideline, with exacerbation of a chronic chest condition being the most prevalent. This proportion varied by time to surgery: from $3.9 \%$ for surgery on the day of admission to $15.5 \%$ for surgery more than 2 days of admission.

\section{Comparison with other studies}

In health services research, the appropriateness of a surgical intervention refers to its expected health benefit exceeding the expected harms by sufficient margin. Originally, measuring the appropriateness of an intervention was motivated by concerns that some patients were not receiving needed treatment. ${ }^{19}$ In hip fracture care, we

Table 2 Prevalence of medically necessary delays to hip fracture surgery among surgically treated patients in relation to timing of admission by timing of surgery

\section{Timing of surgery}

\begin{tabular}{|c|c|c|c|c|}
\hline Timing of admission & Same day & $\begin{array}{l}1 \text { day after } \\
\text { admission }\end{array}$ & $\begin{array}{l}2 \text { days after } \\
\text { admission }\end{array}$ & $\begin{array}{l}\text { More than } 2 \text { days after } \\
\text { admission }\end{array}$ \\
\hline \multicolumn{5}{|c|}{$\%(95 \% \mathrm{Cl})$} \\
\hline Weekday, working hours* & 3.6 (3.2 to 3.9$)$ & 5.0 (4.6 to 5.3$)$ & 7.7 (7.1 to 8.3$)$ & 15.6 (14.7 to 16.6$)$ \\
\hline Weekday, after hours $\dagger$ & 4.1 (3.7 to 4.5$)$ & 4.6 (4.4 to 4.9$)$ & 6.7 (6.3 to 7.1$)$ & 15.4 (14.7 to 16.1$)$ \\
\hline
\end{tabular}

${ }^{*} 08: 00$ to 17:00 on Monday, Tuesday, Wednesday, Thursday or Friday.

†After 17:00 on Monday, Tuesday, Wednesday or Thursday or before 08:00 on Tuesday, Wednesday, Thursday or Friday.

¥Saturday, Sunday or before 08:00 on Monday or after 17:00 on Friday. 
are concerned that real-life care delivery results in some patients not undergoing surgery at the most beneficial time. ${ }^{20}$ Despite the lack of robust evidence about the benefit of early surgery for the wide range of patients seen in clinical practice, physicians and hospitals managers are making decisions every day about patient priority for operating room access. In fact, Lizaur-Utrilla et al argued for preoperative optimisation of the patient's condition with sufficient medical treatment rather than being bound by a general benchmark for timing of surgery. ${ }^{21}$

More information on the underlying reasons for delay is required when determining whether patients benefit from early, or indeed delayed, surgery. Here we assembled a list of diagnosis and procedure codes to identify medical reasons for delay and classified discharge abstracts according to the presence of these codes. In a recent prospective study of 7020 patients, Bretherton and Parker reported that $5.2 \%$ of patients aged 60 years or more, surgically treated for non-pathological hip fracture within 72 hours of admission, presented with at least one NICE 124 condition, which may appropriately delay surgery. ${ }^{22}$ This is comparable to our reported proportion of $5.1 \%$ for patients who underwent surgery within 2 days of admission. We noted an increase in the prevalence of medical reasons for delay on each surgical day since admission. This trend was consistent across admission times, which may suggest robustness of our classification of NICE 124 conditions based on diagnostic and procedure codes.

The proportion of medical reasons for delay varied by patient characteristics. In keeping with previous literature, we found that more men had medical reasons for delay than women. ${ }^{23}$ This may be explained by poorer adherence to medication for chronic conditions for men compared with women. ${ }^{24}$ We also noted more medical reasons for delay among patients admitted from home with comorbidity or home care when compared with those admitted from long-term care. This may suggest poorer control of chronic conditions among older adults in the community, when compared with those supported in long-term care. ${ }^{25}$ Alternatively, this may reflect different coding bias for patients admitted from the community versus long-term care. ${ }^{26}$ In contrast to previous literature, we noted a higher proportion of medical reasons for delay among younger patients when compared with older patients. ${ }^{27}$ The trend reported here may reflect survival bias whereby those with less comorbidities survive into their later years. ${ }^{28}$

\section{Future research}

Here we focused on the feasibility of using administrative data to identify appropriate conditions for delaying hip fracture surgery as listed in the NICE 124 guideline. ${ }^{13}$ However, other medical conditions may also appropriately delay hip fracture surgery. For example, Siegmeth and colleagues included gastrointestinal haemorrhage, uncontrolled hypertension and need for echocardiography. ${ }^{14}$ Deveraux and colleagues proposed an even more extensive list inclusive of frank pulmonary oedema, respiratory failure requiring mechanical ventilation, pulmonary artery hypertension, home oxygen therapy with concomitant clopidogrel, bacteraemia, hereditary or acquired coagulopathy, thrombocytopenia, deep venous thrombosis with vena cava filter, acute stroke, recent subarachnoid haemorrhage, impaired consciousness of unknown origin, new-onset seizure, hyponatraemia, hypokalaemia, hypernatraemia, hyperkalaemia or acidosis. ${ }^{3}$ It can also be argued that patients or their caregivers may choose to delay surgery for personal reasons. ${ }^{29}$ A study assessing the appropriateness of timing of hip fracture surgery at the level of medical history, presentation and test results, as well as personal reasons, is needed to integrate the available evidence with the combined judgement of clinical practitioners and patients and their families to critically evaluate and improve patient selection for early hip fracture surgery.

\section{Limitations}

We identified medical reasons for delay from the ICD-10-CA diagnosis codes and CCI procedures codes present in the CIHI discharge abstracts. The methods are generalisable to other settings that code hospital diagnoses and procedures with ICD-10 and CCI codes. We previously provided code conversions for settings that code diagnoses and procedures with ICD-9 Clinical Modification (ICD-9-CM).$^{30}$ These conversion tables will enable settings that code with ICD-9-CM to implement the methods presented here.

We did not validate the presence of medical reasons for delay in medical records. Our estimate of the proportion of patients requiring anticoagulant reversal is lower than $8 \%$ reported by a study of the UK National Hip Fracture Database. ${ }^{31}$ This may be due to the absence of information relating to medications such as warfarin or clopidogrel in the discharge abstracts. Our estimate of the proportion of patients with anaemia is also lower than the $10 \%$ reported by a recent systematic review. ${ }^{32}$ We selected type 1 (on admission) diagnostic codes only as the timing of postadmission diagnoses were not available, so we could not distinguish between postadmission diagnoses before or after hip fracture surgery. This may have led to an underestimation of the prevalence conditions diagnosed after admission, such as anaemia.

Although procedures, rather than conditions, delay surgery, for some of the NICE conditions, only diagnostic codes were available. This may help to explain why our estimates of the proportion of patients with uncontrolled heart failure, arrhythmia and electrolyte imbalance are also lower than the $3 \%$ reported by chart review. ${ }^{33}$ Our estimates of the proportion of patients with cardiac ischaemia, uncontrolled diabetes and volume depletion are similar to those reported by chart review. ${ }^{33}$ While we estimate that $1 \%$ of patients present with volume depletion, it is likely that this proportion is much higher. ${ }^{33}$ Chronic volume depletion is common in older people secondary to diuretic use and reduced fluid intake. ${ }^{34}$ Standard 
care after hip fracture includes fluid resuscitation from admission to surgery. ${ }^{35}$ Therefore, a formal diagnosis of dehydration or other volume depletion may not be documented in the medical records for coders to abstract. ${ }^{36}$ Using prospective data collection may provide a better vehicle to determine the prevalence of medical, non-medical and personal reasons for delay but would be limited in the number of patients who could be evaluated.

We included codes approved by at least one clinical expert. This is the most conservative rule, which could produce different results as compared with a conclusion from consensus or majority. There were 37 patients without ICD-10-CA codes whom we classified as having no medical delay. We focused on medical reasons for delaying hip fracture surgery. The methods described here may also be applicable outside of hip fracture care. Finally, we identified medical reasons for delay. We did not report non-medical reasons such as resource availability, hospital type or volume, which may contribute to surgical delay.

\section{CONCLUSION}

Administrative data may be used to identify patients presenting with conditions that appropriately delay hip fracture surgery. Accounting for these medically necessary delays may improve estimates of the effectiveness of early surgery. Future research is needed to generate consensus on what constitutes an appropriate medical reason for delay. This will enable improved patient selection for early hip fracture surgery.

\section{Author affiliations}

${ }^{1}$ Centre for Hip Health and Mobility, University of British Columbia, Vancouver,

Canada

${ }^{2}$ Academic Department of Physiotherapy, School of Population Health Sciences,

Kings College London, London, UK

${ }^{3}$ Department of Medicine, McGill University, Montreal, Canada

${ }^{4}$ Division of Orthopaedics, University of Toronto, Toronto, Canada

${ }^{5}$ Division of Orthopaedic Surgery, Dalhousie University, Halifax, Canada

${ }^{6}$ Division of Orthopaedic Surgery, McGill University, Montreal, Canada

${ }^{7}$ Decision Support, Vancouver Coastal Health Authority, Vancouver, Canada

${ }^{8}$ School of Population and Public Health, University of British Columbia, Vancouver, Canada

${ }^{9}$ Vancouver Coastal Health Research Institute, Vancouver, Canada

\section{Twitter @HFStudy}

Acknowledgements The authors acknowledge Jason Kim for generating tables for the manuscript.

Collaborators The following are members of the Canadian Collaborative Study on Hip Fractures: Lauren Beaupre, Eric Bohm, Michael Dunbar, Donald Griesdale, Pierre Guy, Edward Harvey, Erik Hellsten, Susan Jaglal, Hans Kreder, Lisa Kuramoto, Adrian Levy, Suzanne N. Morin, Katie J. Sheehan, Boris Sobolev, Jason M. Sutherland and James Waddell.

Contributors All members of the Canadian Collaborative Study on Hip Fractures contributed to the conception and design of the study. In addition, KJS, BS, MT, LK, SS and PG contributed to the acquisition and the analysis of data. PG, KJS, SNM, JW, MD, EH, SS, BS, LK and MT contributed to the interpretation of the analysis. KJS, BS, LK and MT drafted the manuscript. All members of the Canadian Collaborative Study on Hip Fractures critically revised the manuscript. All members of the Canadian Collaborative Study on Hip Fractures approved the final version for submission.
Funding This research was funded by the Canadian Institute for Health Research (grant number MOP-133629).

Disclaimer The funder has no role in the design of this study, execution, analyses, data interpretation or decision to submit results for publication.

Competing interests The authors declare the following. (1) BS, PG and the Collaborative have received grants from the Canadian Institutes of Health Research related to this work. (2) PG also receives funding from the Natural Sciences and Engineering Research Council of Canada, the Canadian Foundation for Innovation and the British Columbia Specialists Services Committee for work around hip fracture care not related to this manuscript. He has also received fees from the BC Specialists Services Committee (for a provincial quality improvement project on redesign of hip fracture care) and from Stryker Orthopedics (as a product development consultant). He is a board member and shareholder in Traumis Surgical Systems Inc. and a board member for the Canadian Orthopedic Foundation. He also serves on the speakers' bureaus of A0 Trauma North America and Stryker Canada. (3) SM reports research grants from Amgen Canada and from Merck, personal fees from Amgen Canada outside the submitted work.

Ethics approval The University of British Columbia Behavioral Research Ethics Board approved this study.

Provenance and peer review Not commissioned; externally peer reviewed.

Data sharing statement We studied patient records that were anonymised and de-identified by a third party, the Canadian Institute for Health Information, an organization that provides researchers access to data on Canadian residents. Data are available from the Canadian Institute for Health Information for researchers who meet the criteria for access to confidential data.

Open Access This is an Open Access article distributed in accordance with the Creative Commons Attribution Non Commercial (CC BY-NC 4.0) license, which permits others to distribute, remix, adapt, build upon this work non-commercially, and license their derivative works on different terms, provided the original work is properly cited and the use is non-commercial. See: http://creativecommons.org/ licenses/by-nc/4.0/

(c) Article author(s) (or their employer(s) unless otherwise stated in the text of the article) 2017. All rights reserved. No commercial use is permitted unless otherwise expressly granted.

\section{REFERENCES}

1. Centre for Chronic Disease Prevention. Chronic disease and injury framework. 2014 http://infobase.phac-aspc.gc.ca/cdiif/

2. Menzies IB, Mendelson DA, Kates SL, et al. Prevention and clinical management of hip fractures in patients with dementia. Geriatr Orthop Surg Rehabil 2010;1:63-72.

3. Accelerated care versus standard care among patients with hip fracture. The HIP ATTACK pilot trial. CMAJ 2014;186:E52-E60.

4. Beloosesky Y, Grinblat J, Pirotsky A, et al. Different C-reactive protein kinetics in post-operative hip-fractured geriatric patients with and without complications. Gerontology 2004;50:216-22.

5. Beloosesky Y, Hendel D, Weiss A, et al. Cytokines and C-reactive protein production in hip-fracture-operated elderly patients. $J$ Gerontol A Biol Sci Med Sci 2007;62:420-6.

6. Weller I, Wai EK, Jaglal S, et al. The effect of hospital type and surgical delay on mortality after surgery for hip fracture. J Bone Joint Surg Br 2005;87:361-6.

7. Bottle A, Aylin P. Mortality associated with delay in operation after hip fracture: observational study. BMJ 2006;332:947-51.

8. Belmont PJ, Garcia EJ, Romano D, et al. Risk factors for complications and in-hospital mortality following hip fractures: a study using the National Trauma Data Bank. Arch Orthop Trauma Surg 2014;134:597-604.

9. Bergeron E, Lavoie A, Moore L, et al. Is the delay to surgery for isolated hip fracture predictive of outcome in efficient systems? $J$ Trauma 2006;60:753-7.

10. Majumdar SR, Beaupre LA, Johnston DW, et al. Lack of association between mortality and timing of surgical fixation in elderly patients with hip fracture: results of a retrospective population-based cohort study. Med Care 2006;44:552-9.

11. Moran CG, Wenn RT, Sikand M, et al. Early mortality after hip fracture: is delay before surgery important? J Bone Joint Surg Am 2005;87:483-9.

12. Orosz GM, Hannan EL, Magaziner J, et al. Hip fracture in the older patient: reasons for delay in hospitalization and timing of surgical repair. J Am Geriatr Soc 2002;50:1336-40. 
13. National Clinical Guideline Centre. The Management of Hip Fractures in Adults. London: National Clinical Guideline Centre, 2011:53-65. www.ncgc.ac.uk

14. Siegmeth AW, Gurusamy K, Parker MJ. Delay to surgery prolongs hospital stay in patients with fractures of the proximal femur. $J$ Bone Joint Surg Br 2005;87:1123-6.

15. Orosz GM, Magaziner J, Hannan EL, et al. Association of timing of surgery for hip fracture and patient outcomes. JAMA 2004;291:1738-43.

16. Sheehan KJ, Sobolev B, Guy P, et al. Constructing an episode of care from acute hospitalization records for studying effects of timing of hip fracture surgery. J Orthop Res 2016;34:197-204.

17. Canadian Institute for Health Information. Data quality documentation for external users: discharge abstract database, 2010. 2011 https://www.cihi.ca/en/dad_executive_sum_10_11_en. pdf

18. Sobolev B, Guy P, Sheehan KJ, et al. Hospital mortality after hip fracture surgery in relation to length of stay by care delivery factors: A database study. Medicine 2017;96:e6683.

19. Fitch $\mathrm{K}$, et al. The RAND/UCLA appropriateness method user's manual. No. RAND/MR-1269-DG-XII/RE. RAND CORP SANTA MONICA CA. 2001.

20. Lewis PM, Waddell JP. When is the ideal time to operate on a patient with a fracture of the hip? : a review of the available literature. Bone Joint J 2016;98-B:1573-81.

21. Lizaur-Utrilla A, Martinez-Mendez D, Collados-Maestre I, et al. Early surgery within 2 days for hip fracture is not reliable as healthcare quality indicator. Injury 2016;47:1530-5.

22. Bretherton $\mathrm{CP}$, Parker MJ. Early surgery for patients with a fracture of the hip decreases 30-day mortality. Bone Joint J 2015;97-B:104-8.

23. Ricci WM, Brandt A, McAndrew $C$, et al. Factors affecting delay to surgery and length of stay for patients with hip fracture. J Orthop Trauma 2015;29:e109-e114.

24. Hyre AD, Krousel-Wood MA, Muntner P, et al. Prevalence and Predictors of Poor Antihypertensive Medication Adherence in an Urban Health Clinic Setting. J Clin Hypertens 2007;9:179-86.
25. Elliott RA, Goeman D, Beanland C, et al. Ability of older people with dementia or cognitive impairment to manage medicine regimens: a narrative review. Curr Clin Pharmacol 2015;10:213-21.

26. Romano PS, Mark DH. Bias in the coding of hospital discharge data and its implications for quality assessment. Med Care 1994;32:81-90.

27. Fantini MP, Fabbri G, Laus M, et al. Determinants of surgical delay for hip fracture. Surgeon 2011;9:130-4.

28. Lee SJ, Go AS, Lindquist K, et al. Chronic conditions and mortality among the oldest old. Am J Public Health 2008;98:1209-14.

29. Aggarwal A, Harris IA, Naylor JM. Patient preferences for emergency or planned hip fracture surgery: a cross-sectional study. J Orthop Surg Res 2016;11:120.

30. Sheehan KJ, Sobolev B. Guy P For The Canadian Collaborative Study on Hip Fractures, et al. Feasibility of administrative data for studying complications after hip fracture surgery. BMJ Open 2017;7:e015368.

31. Lawrence JE, Fountain DM, Cundall-Curry DJ, et al. Do patients taking warfarin experience delays to theatre, longer hospital stay, and poorer survival after hip fracture? Clin Orthop Relat Res 2017:475:273-9.

32. Potter LJ, Doleman B, Moppett IK. A systematic review of preoperative anaemia and blood transfusion in patients with fractured hips. Anaesthesia 2015;70:483-500.

33. Grimes JP, Gregory PM, Noveck H, et al. The effects of time-tosurgery on mortality and morbidity in patients following hip fracture. Am J Med 2002;112:702-9.

34. Price JD, Sear JW, Venn RM. Perioperative fluid volume optimization following proximal femoral fracture. Cochrane Database Syst Rev 2004:CD003004

35. Moppett IK, Rowlands M, Mannings A, et al. LiDCO-based fluid management in patients undergoing hip fracture surgery under spinal anaesthesia: a randomized trial and systematic review. Br J Anaesth 2015:114:444-59.

36. O'Malley KJ, Cook KF, Price MD, et al. Measuring diagnoses: ICD code accuracy. Health Serv Res 2005;40:1620-39. 